\title{
LICHENS FOUND DURING THE WORCESTER FORAY.
}

\author{
By H. H. Knight, M.A.
}

The woods generally in the Midland Counties have a poor Lichen Flora, and this was the case with those visited during the Foray. Also the northern part of the County may perhaps suffer from the smoke of the industrial towns of the Birmingham district.

In Ockeridge and Shrawley Woods the most noticeable feature was the abundance of Calicium hyperellum and Chaenotheca melanophaea, both growing on oaks. Another of the Caliciaceae, Cyphelium inquinans, was plentiful on old gates outside Trench Woods. Graphidaceae were scarce in all the woods, and Graphis elegans was seen on only one tree. A few saxicole species were noticed on walls by houses, and two common species of Verrucaria were found on Lias stones outside Trench Woods. Specimens of Umbilicaria pustulata gathered on Malvern Hills the previous week were exhibited at the meeting.

$$
\begin{gathered}
W .=\text { Wyre Forest. O.=Ockeridge and Monk Woods. } \\
\text { S. = Shrawley Wood. T.=Trench Woods. }
\end{gathered}
$$

Chaenotheca melanophaea Zwackh. O.S.

Calicium hyperellum Ach. O.S.

Cyphelium inquinans Trev. $T$.

Peltigera canina Willd. S.T.

Parmelia physodes Ach. common.

P. caperata Ach. $S$.

P. saxatilis Ach. $W$.

P. sulcata Tayl. O.S.

P. dubia Schaer. $S$.

P. fuliginosa Nyl. var. laetevirens Nyl. common.

Evernia prunastri Ach. common.

Ramalina fastigiata Ach. $O$.

Usnea florida Web. var. hirta Ach. common.

Xanthoria parietina Th. Fr. on roofs etc.

X. lychnea Th. Fr. $T$.

Candelariella vitellina Müll.-Arg. O.T.

Lecanora subfusca Ach. var, allophana Ach. O.S.

L. campestris B, de Lesd. O.T.

L. galactina Ach. $O$.

L. varia Ach. common.

L. conizaea Nyl. O.S.
L. symmictera Nyl. $T$.

Pertusaria faginea Leight. common.

P. pertusa Dalla Torre and Sarnth. O.S.

P. leioplaca Schaer. O.S.

Phlyctis agelaea Koerb. $S$.

Cladonia fimbriata Fr. W.S. var. subcornuta Nyl. $W$.

C. pityrea Fr. $W$.

C. macilenta Hoffm. $S$.

C. bacillaris Nyl. $W$.

Lecidea ostreata Schaer. W.O.

L. coarctata Nyl. $W$.

L. uliginosa Ach. $S$.

L. parasema Ach. S.T.

Biatorina Griffithii Massal. O.S.T.

Buellia canescens de Not. $T$.

B. myriocarpa Mudd $T$.

Opegrapha herpetica Ach. S.

O. varia Pers. $T$.

$O$. vulgata Ach. $T$.

Graphis elegans Ach. $S$.

Verrucaria nigrescens Pers. $T$.

V. muralis Ach. $T$.

Arthopyrenia fallax Arn. O.S.

Porina carpinea A. Zahlbr. $S$.

Pyrenula nitida Ach. $S$. 